\title{
On the meaning of EPR's Reality Criterion
}

\author{
Márton Gömöri ${ }^{1,2}$ ( Gábor Hofer-Szabó
}

Received: 4 September 2020 / Accepted: 23 August 2021 / Published online: 26 September 2021

(c) The Author(s) 2021

\begin{abstract}
This essay has two main claims about EPR's Reality Criterion. First, we claim that the application of the Reality Criterion makes an essential difference between the EPR argument and Einstein's later arguments against quantum mechanics. We show that while the EPR argument, making use of the Reality Criterion, does derive that certain interpretations of quantum mechanics are incomplete, Einstein's later arguments, making no use of the Reality Criterion, do not prove incompleteness, but rather point to the inadequacy of the Copenhagen interpretation. We take this fact as an indication that the Reality Criterion is a crucial, indispensable component of the incompleteness argument(s). The second claim is more substantive. We argue that the Reality Criterion is a special case of the Common Cause Principle. Finally, we relate this proposal to Tim Maudlin's recent assertion that the Reality Criterion is an analytic truth.
\end{abstract}

Keywords Reality Criterion · EPR argument · Common Cause Principle · Interpretations of quantum mechanics $\cdot$ Incompleteness

\section{Introduction}

Here is how EPR famously formulate in their 1935 paper on the incompleteness of quantum mechanics (QM) what has come to be known as the Reality Criterion (RC):

If, without in any way disturbing a system, we can predict with certainty (i.e. with probability equal to unity) the value of a physical quantity, then there exists an element of physical reality corresponding to this physical quantity. (Einstein et al. 1935, p. 777)

凶 Márton Gömöri

gomorim@gmail.com

Gábor Hofer-Szabó

szabo.gabor@btk.mta.hu

1 Department of Logic, Institute of Philosophy, Eötvös Loránd University, Budapest, Hungary

2 Institute of Philosophy, Research Centre for the Humanities, Budapest, Hungary 
Purely looking at its wording, it is striking how general and deeply philosophical this criterion is. It is a principle of apparent epistemological character that provides a way, as EPR put it, of "recognizing a physical reality" (Einstein et al. 1935, p. 777). Whenever we are in a certain epistemic position, of predicting the result of a measurement without influencing it, we had better adopt a certain ontology. In other words, the RC can be taken as a general inference pattern from the epistemic to the ontic. In this respect it is on a par with Quine (1980)'s and Putnam (1979)'s ideas about ontological commitment based on a successful scientific theory (for one characteristic thing a successful theory provides is a means of predicting measurements without influencing them).

On the other hand, it is remarkable that in the entire history and philosophy of physics-to our knowledge at least-this general epistemological principle has been articulated and applied only once, namely in the EPR argument. No mention of anything like the RC outside of the specific context of the EPR problem in quantum mechanics. Moreover, it is worth observing that the RC is completely lacking from Einstein's later arguments on the incompleteness of quantum mechanics, most of which were explicitly devoted to clarify what the EPR paper had actually been meant to argue. As Fine (1996, p. 5) points out, while the RC plays a central role in the original EPR text, these later arguments no longer make any reference to the criterion; they simply eliminate it.

EPR regarded the $\mathrm{RC}$ as a "reasonable" principle which is "in agreement with classical as well as quantum-mechanical ideas of reality" (Einstein et al. 1935, pp. 777778). It is not entirely clear what Bohr's position was towards the criterion itself, but perhaps it is grounded in his positivistic views that he had doubts about its plausibility, as expressed in his response to the EPR paper (Fine 1996, pp. 34-35). Refining Bohr's subjectivist conception of quantum theory, Quantum Bayesians have come to claim that the RC is an unwarranted principle (Fuchs et al. 2014; Glick and Boge 2019). Even without apparent philosophical commitments, though, most physicists today would probably take it that the RC articulates the kind of "classical realism" that can no longer be maintained in the quantum domain, as is conclusively demonstrated by Bell's theorem (Werner 2014).

Others object. Tim Maudlin (2014) recently argued that the RC is not only a reasonable principle but it is in fact an analytic truth; it is "just not the sort of thing that can coherently be denied," as he writes (Maudlin 2014, p. 7). If there is anything wrong about the EPR argument, or with "classical realism" in general, it is certainly not the $\mathrm{RC}$ to be blamed for it, Maudlin's claim suggests, simply because the criterion lacks any factual content.

Is then the RC a statement without any content? Might this be the reason for the lack of attention to it in the general philosophy of science literature, as well as for the absence of the criterion in later discussions of the EPR problem in Einstein's own writings? Or, rather, does the RC express a substantial philosophical commitment that can be reconsidered and reevaluated-in particular, in light of the results of the EPR-Bell-type experiments? If so, how can Einstein so easily dispense with it in his later reformulations of the EPR idea, while it is a crucial component of the original formulation? 
The aim of this paper is to answer these questions and clarify the meaning of the Criterion of Reality. We believe that the RC articulates a general philosophical principle far from being analytic that can be subject to reconsideration in light of the EPR-Bell problem in quantum mechanics. We will support our view by the following two claims.

First, we will claim that the application of the RC makes an essential difference between the EPR argument and Einstein's later arguments. We will show that while the EPR argument, making use of the RC, does derive that certain interpretations of QM are incomplete, Einstein's later arguments, making no use of the RC, do not prove incompleteness, but rather point to the inadequacy of the Copenhagen interpretation of QM. This suggests that the RC is not eliminable from the incompleteness argument(s)at least from the one(s) we have so far laid out.

The second claim is more substantive. We will claim that the RC is a special case of the Common Cause Principle.

We have to stress at the very outset that our discussion is not indented as a historical analysis. Our primary interest is not what EPR and Einstein actually thought to argue or what they themselves took the RC to be or what interpretation of QM they exactly had in mind. Our analysis is meant to be a logical reconstruction of EPR's and Einstein's arguments, providing a unitary conceptual framework-in terms of causally explaining correlations-to think about these arguments, and to relate them to Bell's treatment and contemporary discussion. To set out such a reconstruction, we will need to sharpen, and to an extent revise, some of our historically loaded terminology-such as what we mean by the Copenhagen interpretation or completeness/incompleteness. In doing so, historical faithfulness and textual details will only be relevant insofar as they provide evidence for the validity of the reconstructed arguments.

In the paper we will proceed as follows. In Sect. 2 we make a distinction between two interpretations of the quantum formalism that will be crucial for our first claim. In Sects. 3, 4 and 5 we address the first claim, that is we compare the EPR argument with Einstein's later arguments. In Sect. 6 we turn to our second claim, namely that the RC is a special case of the Common Cause Principle. In Sect. 7 we relate this proposal to Maudlin's assertion that the Reality Criterion is an analytic truth. We discuss the broader context of our results in Sect. 8.

\section{Two interpretations of the quantum formalism}

Consider the quantum mechanical description of an EPR-Bohm-type scenario, where a pair of spin- $\frac{1}{2}$ particles is emitted by a source and detected at two remote places. The spin state space of the two-particle system is Hilbert space $\mathbb{C}^{2} \otimes \mathbb{C}^{2}$. Suppose that the system is in the singlet state $\Psi_{s}=\frac{1}{\sqrt{2}}\left(\Psi_{+\mathbf{v}} \otimes \Psi_{-\mathbf{v}}-\Psi_{-\mathbf{v}} \otimes \Psi_{+\mathbf{v}}\right)$, where $\Psi_{+\mathbf{v}}, \Psi_{-\mathbf{v}} \in \mathbb{C}^{2}$ are the "up" and "down" eigenvectors of the spin-component operator along an arbitrary direction $\mathbf{v}$. Let $\mathbf{a}$ and $\mathbf{b}$ denote the directions in which the spin of the left and right particle is considered, respectively. We will only be concerned with the "spin up" events in these directions. Let $\hat{A}$ and $\hat{B}$ denote the corresponding projectors on $\mathbb{C}^{2}$, 


$$
\begin{aligned}
& \hat{A}=\left|\Psi_{+\mathbf{a}}\right\rangle\left\langle\Psi_{+\mathbf{a}}\right| \\
& \hat{B}=\left|\Psi_{+\mathbf{b}}\right\rangle\left\langle\Psi_{+\mathbf{b}}\right|
\end{aligned}
$$

and $\hat{I}$ denote the identity on $\mathbb{C}^{2}$. The quantum mechanical description yields the following values for quantum probabilities of single and joint spin outcome events in the given directions:

$$
\begin{aligned}
\left\langle\Psi_{s}, \hat{A} \otimes \hat{I} \Psi_{s}\right\rangle= & \left\langle\Psi_{s}, \hat{I} \otimes \hat{B} \Psi_{s}\right\rangle=\frac{1}{2} \\
& \left\langle\Psi_{s}, \hat{A} \otimes \hat{B} \Psi_{s}\right\rangle=\frac{1}{2} \sin ^{2}\left(\frac{\theta}{2}\right)
\end{aligned}
$$

with $\theta$ being the angle between directions $\mathbf{a}$ and $\mathbf{b}$.

Without further physical interpretation the quantum mechanical description is just a piece of mathematics. The reason is that terms like "state," "event," "quantum probability," while having precise mathematical definitions in the Hilbert space formalism, have no clear physical meaning. For what we shall argue in the following, it will be crucial to distinguish between two different physical interpretations of the formalism of QM. We describe these interpretations on the example of the EPR-Bohm case.

The empirical content of QM is not a matter of interpretative choice. In testing the quantum mechanical description of the EPR-Bohm scenario, quantum probabilities (3)-(4) are compared with long run frequencies of outcomes of spin measurements, performed in an EPR-Bohm-type experiment. If nothing more is assumed, we shall speak about the minimal interpretation of QM.

Minimal interpretation Consider the scheme of an EPR-Bohm-type experiment. A large ensemble of two-particle systems is prepared "in the singlet state" by means of a physical procedure $P_{s}$. In each run of the experiment a member of this ensemble is analyzed: a particle pair is emitted, one particle flying to the left, the other one to the right, and at remote places we measure the spin of the particles along chosen directions. Let $a$ and $b$ denote the events of measuring spin along directions $\mathbf{a}$ and $\mathbf{b}$ in the left and right wings of the experiment respectively. $A$ and $B$ denote the corresponding "spin up" outcomes in the detectors on the two sides. Empirical data are the relative frequencies of measurements $a$ and $b$, and of the corresponding "spin up" outcomes $A$ and $B$, over the ensemble of runs-for varying measurement directions $\mathbf{a}$ and $\mathbf{b}$. On the basis of this observed statistics, one can calculate the conditional probabilities of sort $p\left(A \mid a \wedge P_{S}\right)$, $p\left(B \mid b \wedge P_{S}\right)$, and $p\left(A \wedge B \mid a \wedge b \wedge P_{S}\right)$, defined by Bayes's rule. ${ }^{1}$ The minimal interpretation consists in the identification of quantum probabilities (3)-(4) with these conditional ${ }^{2}$ probabilities of macroscopic measurement events:

\footnotetext{
1 This is not the place to enter into the well-known problem of how probability is precisely related to actual relative frequency. For an overview of the various approaches to probability, see e.g. (Gillies 2000). For a subtle variant of actual frequentism, see (Hoefer 2019).

2 The idea that quantum probabilities are to be interpreted as classical conditional probabilities was first formulated by (Szabó 1995).
} 


$$
\begin{aligned}
\left\langle\Psi_{s}, \hat{A} \otimes \hat{I} \Psi_{s}\right\rangle & =p\left(A \mid a \wedge P_{S}\right) \\
\left\langle\Psi_{s}, \hat{I} \otimes \hat{B} \Psi_{s}\right\rangle & =p\left(B \mid b \wedge P_{S}\right) \\
\left\langle\Psi_{s}, \hat{A} \otimes \hat{B} \Psi_{s}\right\rangle & =p\left(A \wedge B \mid a \wedge b \wedge P_{S}\right)
\end{aligned}
$$

The minimal interpretation only talks about macroscopic measurement events and procedures; in fact, any reference to "particles" or "quantum mechanical system" is dispensable. The quantum mechanical state serves merely as an encryption of the statistics of these visible, macroscopic events. It must be emphasized that since the empirical confirmation of QM is based on the identification (5)-(7), all interpretations of the quantum formalism has to recover it, as long as they are to restore the empirical content of the quantum mechanical description. This is the sense in which the minimal interpretation is "minimal." Different interpretations of the quantum formalism may say different things about what lies behind the macroscopic measurement events; of how "surface" statistics (5)-(7) actually come about.

Next we introduce such an interpretation and dub it Copenhagen interpretation. Since the Copenhagen interpretation is not a monolitic doctrine but a reservoir of various views, we will specify this interpretation by five conditions. As we will see, these conditions will characterize a $\Psi$-ontic collapse theory. We are not concerned here with the historical question whether such a theory can be faithfully attributed to any of the members of the Copenhagen school. Bohr himself did not seem to believe in the collapse since he attributed only a symbolic meaning to the wave function. Von Neuman and Wigner, at the other end of the scale, thought that the reduction is due to the human mind. Maybe the closest to the $\Psi$-ontic collapse theory presented here was Heisenberg who talked about the collapse as an objective physical process (Faye 2019, Sec. 7-8). Be that as it may, we claim that it is the $\Psi$-ontic collapse theory characterized by the following five conditions that was the target of Einstein's later arguments against quantum mechanics. We will see in Sects. 4 and 5 how each of the five conditions below is necessary to derive the contradiction Einstein sets out to establish.

\section{Copenhagen interpretation}

(i) The quantum state not only describes the statistical behavior of an ensemble of systems-encoding, for example, probabilities (5)-(7)-, but is conceived as an element of reality pertaining to the individual system, characterizing every individual member of the ensemble. To distinguish between the quantum state as a mathematical object and the corresponding element of reality, we introduce the following notations:

$\begin{array}{cc}\text { Mathematical object } & \text { Element of reality } \\ \Psi_{s} & \psi_{s} \\ \Psi_{ \pm \mathbf{v}} & \psi_{ \pm \mathbf{v}} \\ \Psi_{ \pm \mathbf{a}} \otimes \Psi_{ \pm \mathbf{b}} & \psi_{ \pm \mathbf{a}} \psi_{ \pm \mathbf{b}}\end{array}$


Accordingly, "big psi" $\Psi$ will denote the wave function, that is a vector of an appropriate Hilbert space, whereas "small psi" $\psi$ will stand for a physical property of a physical system, the property that the wave function represents on the Copenhagen interpretation. In the following we will reserve the term "quantum state" for this physical property. ${ }^{3}$

(ii) Upon performing spin measurements on the particles their quantum state collapses to eigenstates. For example, upon measuring the spin of the right particle in direction $\mathbf{b}$ the singlet state $\psi_{s}$ collapses either to $\psi_{+\mathbf{b}} \psi_{-\mathbf{b}}$ or to $\psi_{-\mathbf{b}} \psi_{+\mathbf{b}}$. Quantum probabilities (3)-(4) are interpreted as the probabilities of collapses resulting in different collapsed states. More precisely:

$$
\begin{aligned}
\left\langle\Psi_{s}, \hat{A} \otimes \hat{I} \Psi_{s}\right\rangle & =p\left(\psi_{+\mathbf{a}} \psi_{-\mathbf{a}} \mid a \wedge \psi_{s}\right) \\
\left\langle\Psi_{s}, \hat{I} \otimes \hat{B} \Psi_{s}\right\rangle & =p\left(\psi_{-\mathbf{b}} \psi_{+\mathbf{b}} \mid b \wedge \psi_{s}\right) \\
\left\langle\Psi_{s}, \hat{A} \otimes \hat{B} \Psi_{s}\right\rangle & =p\left(\psi_{+\mathbf{a}} \psi_{+\mathbf{b}} \mid a \wedge b \wedge \psi_{s}\right)
\end{aligned}
$$

where on the right hand side we have the conditional probabilities of postcollapse quantum states, given the singlet state as a pre-collapse quantum state and spin measurements performed in certain directions.

(iii) If the quantum state of the two-particle system is of form $\psi_{ \pm \mathbf{a}} \psi_{ \pm \mathbf{b}}$ (that is the corresponding element of $\mathbb{C}^{2} \otimes \mathbb{C}^{2}$ has the form of a tensor product $\Psi_{ \pm \mathbf{a}} \otimes \Psi_{ \pm \mathbf{b}}$ ) then the left particle is thought to be in state $\psi_{ \pm \mathbf{a}}$ and the right particle in state $\psi_{ \pm \mathbf{b}}$.

(iv) A particle is attributed "spin up (down) property" along a given direction a if its quantum state is the corresponding eigenstate $\psi_{+\mathbf{a}}\left(\psi_{-\mathbf{a}}\right)$. The operational meaning of this is that if the particle's state collapses to, say, $\psi_{+\mathbf{a}}$ in measurement $a$, then, at the same time, the measurement is assumed to yield the corresponding outcome $A$.

(v) The quantum state $\psi_{ \pm \mathbf{u}}$ not only endows the particle with spin property in direction $\mathbf{u}$ but also determines how the particle is disposed to react to spin measurements in directions $\mathbf{a} \neq \mathbf{u}$, in the sense of predicting probabilities:

$$
p\left(\psi_{+\mathbf{a}} \mid a \wedge \psi_{ \pm \mathbf{u}}\right)=\left\langle\Psi_{+\mathbf{a}}, \hat{A} \Psi_{ \pm \mathbf{u}}\right\rangle
$$

Since in general $p\left(\psi_{+\mathbf{a}} \mid a \wedge \psi_{ \pm \mathbf{u}}\right) \neq p\left(\psi_{+\mathbf{a}} \mid a \wedge \psi_{ \pm \mathbf{v}}\right)$ for $\mathbf{u} \neq \mathbf{v}$, different quantum states will yield different, incompatible predictions for the probabilities of measurement outcomes. Therefore, different quantum states pertain to incompatible elements of reality. That is one can attribute only one quantum state to an individual system at a time. ${ }^{4}$

\footnotetext{
3 This terminology is borrowed from (Maudlin 2019, Ch. 3).

4 The incompatibility of different quantum states is not to be conflated with the incompatibility of noncommuting observables. As we shall see in Sect. 5, while the latter plays no role in Einstein's own incompleteness arguments-as opposed to EPR's original formulation-, the former does play a crucial role in Einstein's considerations. Note that the incompatibility of different quantum states is also an essential ingredient of
} 
The minimal interpretation (5)-(7) is an obvious consequence of collapse probabilities (8)-(10) in conjunction with the operational meaning of collapsed states incorporated in (iii)-(iv). It must be emphasized, however, that the formalism of QM equipped with the minimal interpretation and the formalism of QM equipped with the Copenhagen interpretation are two different theories of the world. As we now turn to discuss, the EPR argument only refers to QM à la the minimal interpretation, whereas Einstein's later arguments are essentially about QM à la the Copenhagen interpretation.

\section{The EPR argument implies the existence of new elements of reality}

Quantum probabilities (3)-(4) in conjunction with the minimal interpretation (5)-(7) yield the following statistics observable in an EPR-Bohm experiment:

$$
\begin{aligned}
p\left(A \mid a \wedge P_{S}\right) & =p\left(B \mid b \wedge P_{S}\right)=\frac{1}{2} \\
p\left(A \wedge B \mid a \wedge b \wedge P_{S}\right) & =\frac{1}{2} \sin ^{2}\left(\frac{\theta}{2}\right)
\end{aligned}
$$

When the measurements in the two wings are performed in the same direction $(\theta=0)$, the outcomes are perfectly anticorrelated:

$$
p\left(A \wedge B \mid a \wedge b \wedge P_{S}\right)=0
$$

That is, if the outcome in one wing is "spin up," the outcome in the other wing is certain to be "spin down," and vice versa. Further, in an EPR-Bohm experiment, events in the two wings-the tokens of $a, A$ and $b, B$ respectively-are spatially separated in each run. Consequently, assuming relativistic causality, what happens in one wing cannot have influence on what happens in the other wing. Then, in a given run of the experiment where parallel measurement directions have been chosen, the premises of the RC fulfill: on the basis of performing spin measurement in the right wing, without influencing the outcome in the left wing, we can be sure about the outcome of spin measurement in the left wing (and vice versa). That is to say, "without in any way disturbing a system, we can predict with certainty (i.e. with probability equal to unity) the value of a physical quantity." 5 If the RC is true, then "there exists an element of physical reality corresponding to this physical quantity."

What does it take to be an element of physical reality corresponding to a physical quantity? More precisely, how does an element of reality corresponding to a physical quantity relate to those elements of reality that correspond to the measurement of

\footnotetext{
Footnote 4 Continued

Harrigan and Spekkens (2010)(p. 125) definition of a $\Psi$-ontic theory: "We call a hidden variable model $\Psi$-ontic if every complete physical state or ontic state in the theory is consistent with only one pure quantum state."

5 In this context, we need not distinguish between the value of a physical quantity and the outcome of a measurement (cf. Healey 2017, p. 101). In fact, as it will be clear from Sect. 6 where the RC will be proved to be a special case of a much more general principle, the subject of our prediction in the formula of the $\mathrm{RC}$ can be any physical event related to the system in question.
} 
this quantity, to the elements of reality the minimal interpretation refers to? Here is Goldstein et al. (2011) pointing out what the "element of physical reality" in the RC essentially does:

This criterion [the RC] simply reflects the fact that if the outcome of some experiment isn't pre-determined by some element of physical reality (i.e., if it is not a function of something that was an element of physical reality before the experiment) then its outcome involves some randomness and hence cannot be predicted with certainty. (Goldstein et al. 2011, Sec. 10.3)

That is, an element of reality corresponding to a physical quantity is something that determines the outcome of measurement whenever the value of the quantity in question is measured. Consequently, according to the RC, in each run of the EPR-Bohm experiment where parallel measurement directions are chosen, the results of spin measurements in both ${ }^{6}$ wings must be predetermined.

Notice that the above reflection of Goldstein et al. does not make any reference to the clause of the RC "without in any way disturbing a system." Indeed, if I am able to predict with certainty the outcome of a measurement, even if by means of disturbing the system, then this outcome must in some way be predetermined, otherwise, the point of Goldstein et al. being still valid, it could not be predicted with certainty. Nevertheless, if the act of my "prediction" can influence the outcome, then the element of reality that determines this outcome may well be the very act of my "prediction." That is, it may be the performance of measurement in the right wing that determines the outcome in the left wing. ${ }^{7}$ If, however, the events in the two wings are spatially separated, and thereby the act of prediction can no longer influence, and hence determine, the predicted outcome, then this possibility is excluded. In this case the RC implies the presence of new elements of reality that are not present according to the minimal interpretation of the quantum mechanical description. ${ }^{8}$

The RC only guarantees the outcomes of spin measurements to be predetermined when the measurements in the two wings are performed in the same direction. This is because for $\theta \neq 0$ the probabilities (12)-(13) imply non-perfect correlation that does not allow for predicting with certainty the outcome in one wing on the basis of the outcome in the other wing; and hence the RC is not applicable. Moreover, even when the same measurement directions are chosen in the two wings, the RC only predicts the presence of those elements of reality that correspond to spin values in this chosen direction, but not in any other direction. However, the element of reality idea has another aspect, not reflected in the above quote by Goldstein et al., ensuring that

\footnotetext{
${ }^{6}$ On the face of it, the statement of the RC, as applied to the EPR-Bohm situation, is asymmetric with respect to the outcomes in the two wings, in that we pick one of them to predict the other. But of course nothing hings on which outcome is selected for which role, so the consequent of the RC, the fact that the outcome in question is predetermined, must hold for both outcomes.

7 In connection with footnote 6 , note that in this case the RC cannot be applied to infer that the right outcome, used here as a means of "predicting" the left outcome, is also predetermined (in fact it can be fully random). For if the right outcome is causally informed by the left measurement then the former cannot be reasonably regarded as a prediction of the latter. See also the discussion in Sect. 6 on how the RC's terms translate to a causal-statistical language.

${ }^{8}$ Nor they are present according to the Copenhagen interpretation. This is because none of the spin outcomes are predetermined in the singlet state.
} 
in fact all spin outcomes in all directions must be predetermined in each run of the experiment. Here is EPR considering this problem:

One could object to this conclusion [that the spin outcomes are predetermined in all directions] on the grounds that our criterion of reality is not sufficiently restrictive. Indeed, one would not arrive at our conclusion if one insisted that two or more physical quantities can be regarded as simultaneous elements of reality only when they can be simultaneously measured or predicted. On this point of view, since either one or the other, but not both simultaneously, of the quantities [of the spin along directions $\mathbf{v}$ and $\mathbf{v}^{\prime}$ ] can be predicted, they are not simultaneously real. This makes the reality of [the spin of the second system along directions $\mathbf{v}$ and $\mathbf{v}^{\prime}$ ] depend upon the process of measurement carried out on the first system, which does not disturb the second system in any way. No reasonable definition of reality could be expected to permit this. (Einstein et al. 1935, p. 780)

EPR refer to the spatial separation of events in the two wings. They point out that if the element of reality that determines the outcome in one wing is, in each run of the experiment, assumed to be localized in the same wing, then the presence of this element of reality cannot depend on the direction in which we choose to measure in the other wing due to the causal separation of the wings. Note that the absence of direct causal influence between the measurement choice in one wing and the elements of reality in the other wing does not itself mean that there can be no statistical dependence (even maximal correlation) between the two, brought about by a "common cause." This possibility is excluded not by locality considerations but by an independent assumption, as is emphasized by Goldstein et al.:

Even though, in each run of the experiment, either the $z$-axis or the $x$-axis is chosen along which to perform the measurements, the elements of physical reality that exist before the measurements cannot depend on choices that will be made later by the experimenters! This, indeed, doesn't follow from the assumption of locality itself but it does follow from the so-called "no conspiracy" assumption which states, roughly speaking, that the pair of particles prepared by the source does not "know" in advance what experiments are going to be performed on them later. (Goldstein et al. 2011, Sec. 10.3)

Of course not only it is assumed that the particles do not "know" what experiments are going to be performed on them, but also that the experimenters do not "know" what elements of reality, corresponding to the spin values of the particles along different directions, are present, and hence their choice of the measurement setup does not depend on these elements of reality. It is assumed that the presence of the elements of reality that predetermine the outcomes and the choices of measurements are influenced by different factors, and therefore there exists no common factor that could influence them both. The absence of common cause, in conjunction with the absence of direct causal connection following from locality, ${ }^{9}$ together imply that there is nothing that

\footnotetext{
9 Some caution is in order here. Notice that the direction in which the spin outcome is guaranteed to be determined, as a consequence of the RC, not only depends on the remote measurement choice but also on
} 
could serve to correlate the measurement choices and the elements of reality in question, and hence their distributions over the runs of the experiment must be statistically independent. This in turn will ensure EPR's conclusion: there must exist elements of reality corresponding to the spin values of the two particles in all directions in all runs of the experiment. ${ }^{10}$

\section{Einstein's later arguments imply that the wave function cannot represent an element of reality pertaining to the individual system}

It must be emphasized that the EPR argument can be formulated merely in terms of the minimal interpretation of QM. Therefore, the argument is independent of which interpretation $^{11}$ of QM one may favor. It is independent of any assumption expressed in terms of what may lie behind the macroscopic measurement events. ${ }^{12}$

In contrast, EPR's original formulation does employ a specific interpretation, namely the Copenhagen interpretation. Thus, one may not be surprised that Einstein himself was dissatisfied with the EPR text, saying "the main point was, so to speak, buried by the erudition" (Howard 1985, p. 175). However, what Einstein took to be the main point of the EPR idea is rather different, much simpler, then what we have

\section{Footnote 9 Continued}

the local one; for the presence of the element of reality corresponding to the left spin in direction $\mathbf{v}$ only follows if the measurements in both of the two wings are performed along $\mathbf{v}$. If the element of reality determining the spin outcome on the left is assumed to be localized in the left wing (one can identify the "left wing" with the past light cone of the left outcome), without more specific constraints on its localization, then it seems possible that this element of reality is affected by the local measurement choice, through the process of measurement performed in the left wing. Such an influence could break down the independence of the measurement choices and the elements of reality in question. That the element of reality must be fixed before the local measurement choice is made, excluding the possibility of such influence, as is suggested by Goldstein et al. above, is something that is not implied by what has been said so far about the RC. We will see how this condition follows in Sect. 6 (footnote 23) where we discuss the deeper meaning of the RC.

10 Indeed, let $\lambda$ denote the element of reality that predetermines the outcomes of spin measurements along an arbitrary given direction $\mathbf{v}$ in the two wings. The requirement that $\lambda$ be statistically independent from the choices of measurements just means

$$
p(\lambda \mid c)=p(\lambda)
$$

where $c$ denotes an arbitrary Boolean combination of measurement events $a$ and $b$. Consider the subensemble of runs where the measurement direction is set to be $\mathbf{v}$ in both wings $(\mathbf{a}=\mathbf{b}=\mathbf{v})$. In this subensemble the outcomes must be predetermined and the element of reality $\lambda$ is certain to be present:

$$
p(\lambda \mid a \wedge b)=1
$$

Now apply (15) to $c=a \wedge b$ to obtain

$$
p(\lambda)=1
$$

which is to say that the outcomes of spin measurements along the arbitrary direction $\mathbf{v}$ are predetermined in the whole ensemble, that is in all runs of the experiment.

11 One exception is the many-worlds interpretation which circumvents the conclusion of EPR by refuting that correlation (14) is real. Cf. (Maudlin 2014, pp. 22-23).

12 For example, such an assumption is the "separability" of quantum systems (Howard 1985). 
outlined in the previous section, and is indeed strongly related to the Copenhagen interpretation. The seed of this thought is there in the EPR paper:

[...] as a consequence of two different measurements performed upon the first system, the second system may be left in states with two different wave functions. On the other hand, since at the time of measurement the two systems no longer interact, no real change can take place in the second system in consequence of anything that may be done to the first system. This is, of course, merely a statement of what is meant by the absence of an interaction between the two systems. Thus, it is possible to assign two different wave functions [...] to the same reality (the second system after the interaction with the first). (Einstein et al. 1935, p. 779)

After the EPR paper Einstein formulated his worries about quantum mechanics in various different versions-among them in a letter to Schrödinger (dated June 19, 1935); in a letter to Popper (dated September 11, 1935); in his 1936 "Physics and Reality" paper (Einstein 1936); and in his "Autobiographical Notes" in 1948 (Einstein 1948). ${ }^{13}$ All these considerations are variations and elaborations of the same theme voiced in the above passage by EPR. What these reflections share is the adoption of the quantum state collapse idea and, as Fine (1996, p. 5) stresses, the overall absence of any reference to the RC that was so crucial to EPR. Here is Einstein's 1936 argument:

Consider a mechanical system consisting of two partial systems $A$ and $B$ which interact with each other only during a limited time. Let the $\Psi$ function before their interaction be given. Then the Schrödinger equation will furnish the $\Psi$ function after the interaction has taken place. Let us now determine the physical state of the partial system $A$ as completely as possible by measurements. Then quantum mechanics allows us to determine the $\Psi$ function of the partial system $B$ from the measurements made, and from the $\Psi$ function of the total system. This determination, however, gives a result which depends upon which of the physical quantities (observables) of $A$ have been measured (for instance, coordinates or momenta). Since there can be only one physical state of $B$ after the interaction which cannot reasonably be considered to depend on the particular measurement we perform on the system $A$ separated from $B$ it may be concluded that the $\Psi$ function is not unambiguously coordinated to the physical state. This coordination of several $\Psi$ functions to the same physical state of system $B$ shows again that the $\Psi$ function cannot be interpreted as a (complete) description of a physical state of a single system. (Einstein 1936, [1936] 1954, p. 317)

The essence of Einstein's observation is that the quantum state collapse idea involved in the Copenhagen interpretation is in contradiction with the physical/causal separation of the partial systems (that is, with locality if causal separation is ensured by the spatial separation of the two systems). For the sake of concreteness, let us reconstruct this argument as applied to the EPR-Bohm scenario. On the Copenhagen interpretation, in every run of the EPR-Bohm experiment where first the right wing measurement $b$

\footnotetext{
13 For the various reconstructions of the EPR argument and Einstein's later arguments and many related topics see e.g. (Howard 1985), (Redhead 1987, Ch. 3), (Hájek and Bub 1992), (Fine 1996, Ch. 3), (Norton 2018), and (Harrigan and Spekkens 2010).
} 
is performed, the singlet state $\psi_{s}$ collapses to $\psi_{+\mathbf{b}} \psi_{-\mathbf{b}}$ or to $\psi_{-\mathbf{b}} \psi_{+\mathbf{b}}$ (clause (ii) in Sect. 2). According to the ontology of the Copenhagen interpretation, in these runs, after the measurement on the right has been performed, the particle on the left comes to be in state $\psi_{ \pm \mathbf{b}}$; meaning that there is an element of reality localized in the left wing that corresponds to the particle being in that state (clause (iii) in Sect. 2). Since $\psi_{ \pm \mathbf{b}}$ is a function of $\mathbf{b}$, this element of reality is dependent on which one of the measurement directions has been selected in the other wing. How to interpret this functional dependence? It is clear from Einstein's words that what he has in mind is a sort of counterfactual dependence. But if for any reason one wants to avoid reference to counterfactuals, there is no need to employ these terms. For what functional dependence certainly implies is statistical correlation over the runs of the experiment. ${ }^{14}$ Now, due to locality, this correlation of the element of reality in one wing and the measurement choice made in the other wing, cannot be a consequence of direct casual connection, since the two wings are spatially separated. The absence of direct casual dependence, in itself, does not mean that the two events in question cannot be dependent in a statistical sense, for there may exist a "common cause" that brings about correlation. However, this possibility is again excluded by no-conspiracy: if the measurement choice $b$ is assumed to be autonomous, for example due to its being governed by a local random number generator, then $b$ cannot be affected by anything that influences the state of affairs in the left wing, including the quantum state of the left particle. All in all, the element of reality the Copenhagen interpretation assumes to exist in the left wing, $\psi_{ \pm \mathbf{b}}$, cannot be brought about by any conceivable causal means. Therefore, contrary to what the Copenhagen interpretation postulates, the wave function $\Psi_{ \pm \mathbf{b}}$ cannot represent an element of reality $\psi_{ \pm \mathbf{b}}$ characterizing the physical state of the individual particles. In other words, "the $\Psi$ function cannot be interpreted as a [...] description of a physical state of a single system."

The conclusion of Einstein's argument is that according to the Copenhagen interpretation there ought to exist elements of reality, corresponding to the quantum state of the individual particles after state collapse, that cannot exist under the assumptions of locality and no-conspiracy.

\section{The EPR argument is an incompleteness argument; Einstein's later arguments are not}

The EPR argument and Einstein's later arguments are different in their premises and different in their conclusions. More precisely, they are different to the extent that:

14 The only way $\psi_{s}$ can collapse to $\psi_{ \pm \mathbf{b}} \psi_{\mp \mathbf{b}}$ is when the measurement choice in the right wing is $b$. This implies correlation between the collapsed quantum state and the right wing measurement choice:

$$
\frac{1}{2}=p\left(\psi_{ \pm \mathbf{b}} \psi_{\mp \mathbf{b}} \mid b \wedge \psi_{S}\right)>p\left(\psi_{ \pm \mathbf{b}} \psi_{\mp \mathbf{b}} \mid \neg b \wedge \psi_{s}\right)=0
$$

given that $0<p(b), p(\neg b)<1$, where $\neg b$ denotes the "negation" of $b$, more precisely, the event of choosing an arbitrary measurement direction in the right wing other than $\mathbf{b}$. 
(a) The EPR argument can be formulated in terms of the minimal interpretation of QM, whereas Einstein's later arguments are essentially tied to the Copenhagen interpretation.

(b) The crucial ingredients of the EPR argument are locality, no-conspiracy, the perfect correlation predicted by (the minimal interpretation of) QM, and the RC. In contrast, Einstein's later arguments make no reference to perfect correlation, neither to the RC; their essence is to point to the tension between the quantum state collapse idea and locality (in conjunction with no-conspiracy).

(c) The EPR argument implies the existence of elements of reality not described by QM, neither on the the minimal interpretation, nor on the Copenhagen interpretation. Thus, QM, on either of these interpretations, is incomplete. According to Einstein's later argument, the Copenhagen interpretation is committed to the existence of elements of reality that cannot be out there in the world-under the assumptions of locality and no-conspiracy. Hence, given these assumptions, the Copenhagen interpretation is unsound-as opposed to being incomplete.

Despite these substantial differences the EPR and Einstein's later arguments are often lumped together in the literature: both of them are labeled as "incompleteness argument." This terminology also finds support in Einstein's writings. Einstein saw his own reflections on the subject as refinements of the EPR idea purporting to point to the fundamental incompleteness of QM. ${ }^{15}$ As remark (c) indicates this terminology is not quite apt nonetheless. Many commentators have noticed (Hájek and Bub 1992, p. 327; 1985, pp. 180-181; 2014, pp. 334-336) that the condition Einstein establishes in his 1936 argument (as well as in all of his similar arguments)-“the $\Psi$ function is not unambiguously coordinated to the physical state"-is not identical with, but just the converse of the condition of incompleteness. EPR (Einstein et al. 1935, p. 777) define completeness as "every element of the physical reality must have a counterpart in the physical theory.") Lehner (2014) even introduces a terminology to mark this difference: he saves "incompleteness" for EPR's condition, while using "overcompleteness" for Einstein's condition. On the basis of this distinction, Lehrer (2014, p. 337) and (Uffink 2020, p. 559) point out that Einstein's own reasoning provides a weaker argument for incompleteness than the EPR argument. However, this way of putting it still obscures the essential difference between Einstein's and EPR's target. If a theory is "overcomplete" in the sense Einstein argues Copenhagen QM must be, then it postulates more elements of reality then what there is in the world. Consequently, some of the theory's ontological claims are plainly false. Such a description already has a name: it is inadequate or unsound. Note that this latter terminology is in complete harmony with the way (semantic) "completeness" and "soundness" is used in logic (though not for the characterization of theories but of logical systems). "Completeness" is used when everything that is true in the world (in the model) is also stated by the theory; "soundness" is used when there is no statement of the theory that is not true in the world. In accord with this standard terminology, Einstein's later arguments are better be characterized as unsoundness arguments, rather than incompleteness arguments. ${ }^{16}$

\footnotetext{
15 But there is also indication that Einstein's view about the relation of his later arguments and the completeness question is subtler than this. See below in this section.

16 Note that drawing parallel between the problem of completeness in quantum mechanics and in logic is not unprecedented in the literature. Howard and Giovanelli (2019) argue that the notion of completeness in
} 
Since many may find this conclusion controversial, it will be useful to compare our discussion with other reconstructions of Einstein's later arguments in the literature. Howard (1985, p. 181) offers an interesting reasoning about the way to see Einstein's argument as pointing to incompleteness:

'Completeness' surely requires of the $\Psi$-function that it describe all of the properties of a system in a given real state, so that different real states must be described by different $\Psi$-functions (which is all that the [...] EPR completeness condition demands). But why require, conversely, that different $\Psi$-functions always be correlated with different real states? Might there not be situations in which the differences between two $\Psi$-functions (phase differences, for example) are inessential from the point of view of the system whose real state they aim to describe? Einstein's completeness condition would, indeed, be too strong if it required that literally every difference between $\Psi$-functions mirror a difference in the real state of the system in question; but such was not Einstein's intention. The kind of difference with which Einstein was concerned is clear from his argument: $\Psi_{B}$ and $\Psi_{\bar{B}}$ [two different wave functions of system $B$ left behind after performing one of two alternative measurements on system $A$ ] differ in the predictions they yield for the results of certain objective, local measurements on system $B$. This is not an inessential difference, and Einstein was right in holding that it is excluded by the demand for completeness, for the only way to account for such a difference (assuming that both $\Psi$-functions provide 'correct' descriptions) is by assuming that at least one of the two $\Psi$-functions, or perhaps each of them, gives an incomplete description. [...] Two different $\Psi$-functions can give correct descriptions of one and the same real state only if one of them, at least, tells less than the whole truth about that real state.

Howard thinks there is a way to avoid concluding that the Copenhagen interpretation is unsound, namely by assuming that it is incomplete. We believe, however, that there is no such a way out. It is precisely because " $\Psi_{B}$ and $\Psi_{\bar{B}}$ differ in the predictions they yield for the results of certain objective, local measurements on system $B$," that the two wave functions are incompatible, and so they cannot be assigned to the same real state by any correct theory of the world, even if it only provides an incomplete description. $^{17}$

The incompatibility of different quantum states-we formulated it as an ingredient of the Copenhagen interpretation in clause (v) in Sect. 2-was in fact a crucial assumption in our own reconstruction of Einstein's argument too. For if one could coherently assign more than one quantum state, the corresponding element of reality, to an individual system, then one could also coherently imagine that the particle in the left wing in fact occupies one of the spin eigenstates for every spatial direction at the same time. In other words, one could imagine that the physical state of the

Footnote 16 Continued

Einstein's own arguments is analogous to, and in fact originates in, the concept of categoricity as it is used in model theory.

17 By analogy, suppose that a theory claims that a die is both fair and biased at the same time. Now, you will not be able to avoid the conclusion that this theory is wrong by acknowledging that both "fair" and "biased" are incomplete descriptions of the (rolling of the) die. 
left particle, after the right wing measurement has been carried out, simultaneously incorporates $\psi_{+\mathbf{v}}$ or $\psi_{-\mathbf{v}}$ for all $\mathbf{v}$, not only the one that results after collapsing $\psi_{s}$ by the measurement $b$ actually performed. Then the physical state of the particle, incorporating all these $\psi_{ \pm \mathbf{v}}$-s as partial descriptions, in fact would not be dependent on the measurement choice in the other wing-the state collapse would not bring about a correlation between the physical state of the left particle and the performance of the right wing measurement. ${ }^{18}$ Thus, one would have no contradiction with the causal separation of the two wings ensured by locality and no-conspiracy in Einstein's argument. So if different quantum states were compatible elements of reality, then giving up seeing them as complete descriptions would indeed provide a way out from concluding that they must be incorrect descriptions-just as Howard suggests. But, again, because different quantum states yield incompatible probability predictions, they cannot simultaneously provide correct descriptions, even if each of them provides only an incomplete description. ${ }^{19}$ Hence, incompleteness is not an antidote to the inadequacy of the Copenhagen interpretation.

Harrigan and Spekkens (2010) recently argued for a thesis that resonates with our conclusion. Here is how they summarize their result:

What is not typically recognized, and which we show explicitly here, is that the latter argument was actually strong enough to also rule out locality for $\Psi$-ontic hidden variable theories. In other words, Einstein showed that not only is locality inconsistent with $\Psi$ being a complete description of reality, it is also inconsistent with $\Psi$ being ontic, that is, inconsistent with the notion that $\Psi$ represents reality even in an incomplete sense. Einstein thus provided an argument for the epistemic character of $\Psi$ based on locality. (Harrigan and Spekkens 2010, pp. 126-127)

Indeed, since the Copenhagen interpretation is $\Psi$-ontic (that is it takes $\Psi$ to represent an element of reality pertaining to the individual system), what Einstein must have achieved, based on the locality assumption, is proving it unsound, rather than incomplete. However, Harrigan and Spekkens suggest that what Einstein actually showed is stronger than incompleteness, hence his argument still entails incompleteness. This suggestion is made explicit on p. 133 (Lemma 6) where they formulate the claim that the non-ontic ("epistemic") character of $\Psi$ entails its incompleteness. Notice, however, the important difference between the notion that $\Psi$, whether on an ontic or epistemic interpretation, alone provides an incomplete description of reality, and the notion that the theory which features $\Psi$, QM under a certain interpretation, as a whole is incomplete. Clearly, Harrigan and Spekkens's notion of " $\Psi$-incompleteness" (Definition 2-3, p. 131) corresponds to the first conception, while EPR's incompleteness refers to a theory and hence it corresponds to the second one. The non-ontic character of $\Psi$ evidently implies $\Psi$-incompleteness, for if $\Psi$ doesn't represent an element of reality (pertaining to the individual system) at all, then, a fortiori, it cannot represent all elements of reality (pertaining to the individual system) either-as completeness would

\footnotetext{
18 The right hand side of (18) would no longer be forced to equal to 0 .

19 Notice the difference here between the EPR and Einstein's arguments. EPR infer, through the RC, that the particles posses spin properties in all directions. By contrast, we are not entitled to ascribe to the particles, through state collapse, all quantum states $\psi_{+\mathbf{v}}$ or $\psi_{-\mathbf{v}}$ for all directions $\mathbf{v}$. This is because while spin properties in different directions are logically compatible, quantum states are not.
} 
require. But $\Psi$-incompleteness is entirely silent about whether the theory which features $\Psi$, as a whole, is complete or not. A case in point is the minimal interpretation of QM. QM à la the minimal interpretation, as we defined it in Sect. 2, is certainly a non- $\Psi$-ontic theory, and hence it is also $\Psi$-incomplete. But the mere fact of this doesn't entail that QM à la the minimal interpretation must be an incomplete theory. ${ }^{20}$ Indeed, to show that, we need the EPR argument. Thus, while Harrigan and Spekkens are right to state that Einstein's argument rules out the $\Psi$-complete interpretations of QM, this doesn't make Einstein's argument an incompleteness argument, in the sense of EPR's condition of completeness. For, there is no particular account of reality that is shown to be incomplete by Einstein's argument. What the argument shows is that the $\Psi$-ontic account of quantum phenomena provided by the Copenhagen interpretation of QM is unsound.

Note, again, the sense in which Einstein's conclusion entails $\Psi$-incompleteness: if $\Psi$ cannot be interpreted as the description of the physical state of a single system, then it certainly cannot provide a complete description of such a state. This is just what Einstein seems to imply, in the last quoted sentence of his 1936 argument above, by putting the term "complete" in parenthesis. The almost off-hand manner in which Einstein mentions completeness seems to be an indication that the focus of his reasoning is not so much the incompleteness of the wave function, but the very fact that it cannot be regarded as a description of individual systems at all. Einstein seems to have been fully aware of the distinction between $\Psi$-incompleteness, as it is established by his own argument, and the notion that quantum theory is incomplete, as it is defined and defended it in the EPR paper. A clear evidence of this is the way he actually relates his finding about the non-ontic character of the wave function to the question of completeness in a later part of the 1936 paper-a way which is significantly different from the off-handed reference to $\Psi$-incompleteness in the quoted sentence. To see this relation, let us follow the line of his 1936 reasoning. To the quoted passage of the 1936 paper Einstein immediately adds: "Here also the coordination of the $\Psi$ function to an ensemble of systems eliminates every difficulty. A measurement on $A$, for example, thus involves a transition to a narrower ensemble of systems. The latter (hence also its $\Psi$ function) depends upon the point of view according to which this reduction of the ensemble of systems is carried out." (Einstein 1936, [1936] 1954, p. 317) Einstein suggests that the tension of locality and wave function collapse is resolvable by abandoning the Copenhagen interpretation and adopting what might be called an ensemble interpretation of the wave function. It is not completely clear what exactly Einstein's ensemble interpretation might have been (cf. Fine 1996, Ch. 4), but what is certainly involved in it is the insistence that the wave function, rather than pertaining to the ontology of the single system, merely serves as an encryption of statistical features of an ensemble of systems. On the other hand, while adopting an ensemble interpretation naturally resolves the problem raised by his later arguments,

\footnotetext{
20 Recall that the elements of reality posited by the minimal interpretation of QM are macroscopic measurement events such as preparation and measurement procedures and outcomes of measurements. There do exist approaches to quantum theory holding that QM expressed in these macroscopic terms should be regarded as complete. An example of such an approach is the so-called operational QM advocated by (Werner 2014).
} 
Einstein did not believe that a merely statistical account of quantum phenomena could be regarded as a complete description. In his words:

What happens to the single system remains, it is true, entirely unclarified by this mode of consideration; this enigmatic happening is entirely eliminated from the representation by the statistical manner of consideration.

But now I ask: Is there really any physicist who believes that we shall never get any inside view of these important alterations in the single systems, in their structure and their causal connections, and this regardless of the fact that these single happenings have been brought so close to us, thanks to the marvelous inventions of the Wilson chamber and the Geiger counter? To believe this is logically possible without contradiction; but, it is so very contrary to my scientific instinct that I cannot forego the search for a more complete conception. (Einstein 1936, [1936] 1954, p. 318)

Thus, it is not Einstein's later arguments themselves, directed against the Copenhagen interpretation, that imply incompleteness (in EPR's sense of the term); rather it is the natural response to these arguments, the adoption of an ensemble interpretation, that suggests, rather than implies, the EPR-incompleteness of QM (à la an ensemble interpretation).

To conclude: Einstein's later arguments are not incompleteness arguments. It is only the EPR argument that implies the incompleteness of QM. The EPR argument points to a particular type of element of reality that is not described by QM, but which, according to the premises of the argument, should nonetheless be out there in the physical world. What makes it possible to infer the existence of elements of reality out there in the world? It is the RC that renders it possible, without which no new elements of reality are inferred. The RC is thus not eliminable from the incompleteness argument(s)-at least from the one(s) we have so far laid out. This suggest that it must have a substantial content. Now we turn to the question of the precise meaning of this principle.

\section{The Reality Criterion is a special case of the Common Cause Principle}

Consider again how Goldstein et al. (2011) formulates the essence of the RC:

This criterion simply reflects the fact that if the outcome of some experiment isn't pre-determined by some element of physical reality [...] then its outcome involves some randomness and hence cannot be predicted with certainty. (Goldstein et al. 2011, Sec. 10.3)

What, exactly, is the source of the impossibility that the RC associates with the prediction of a random outcome? Suppose you flip a coin a thousand times. Suppose that your friend, every time you flip the coin, makes a guess about the result, before it is revealed. Imagine that she always gets it right: whenever she predicts Heads, the coin comes up Heads, whenever she predicts Tails, the coin comes up Tails. Such a coincidence would cry out for an explanation. Either your friend's prediction must somehow influence the outcome, for example by you altering the coin's position before revealing it so as 
to match her guess; or her prediction must, in some way, be informed by the flip of the coin, for example by her employing a computer that detects the mechanical initial data of the coin flip and calculates the outcome on the basis of the laws of mechanics. In either case, the outcome must be predetermined-by your friend's guess or by the initial conditions of the coin flip-, otherwise the perfect match of the guesses with the outcomes would be a magical coincidence, a correlation without causal explanation. Thus, the RC, we believe, is nothing but the expression of the intuition that correlations-in particular, the correlation between the act of predicting something on the one hand and the predicted phenomena on the other-require explanation in terms of causal connections. That is, the RC is just a special case of the Common Cause Principle (CCP). This claim is what we are going to expand on and argue for in the following.

To make this claim precise, we need to have a translation between the RC's terms and the language of the CCP. First, let us make explicit the conceptual framework in which both these principles will be formulated. Consider the statistical ensemble of the runs of an experiment performed repeatedly for a large number of times. Each run is a token event that may instantiate certain event types. For example, let $a$ denote the event type of performing a given measurement and let $A$ denote the event type of one of its outcome occurring. Consider the statistics of the different event types over the ensemble of runs. Let $p(a), p(A), p(A \mid a)$, etc. denote the corresponding probabilities. In each individual run the token events can be causally related. Let $a \rightarrow A$ denote that the tokens of $a$ have, at least in some runs of the experiment, causal influence on the tokens of $A$ ( $\nrightarrow$ will denote the absence of casual influence). We will not be concerned here about the meaning of this causal relation. We will see, however, that both the RC's and the CCP's statements presuppose that we can meaningfully talk about such a relation.

Let us now try to unpack the RC's statement in the above terms. What do we mean by "predicting with certainty (i.e. with probability equal to unity) the value of a physical quantity"? Let $a$ denote the measurement of the quantity in question and $A$ be the outcome event corresponding to the quantity having a certain value. The act of predicting this quantity is an event (in the coin example, your friend uttering, say, "Heads"). Let $B$ denote this event: predicting that the quantity in question has a value pertaining to outcome $A$. The prediction is certain if whenever $B$ happens then, given that $a$ is performed, the measurement results in outcome $A$. That is:

$$
p(A \mid a \wedge B)=1
$$

Event $B$ only qualifies as a prediction if $B$ happens before outcome $A$ is revealed; more precisely, if $A$ doesn't causally precede $B$, meaning that the act of prediction is not influenced by the outcome to be predicted. (If your friend sees the outcome of the coin flip before "predicting" it, then it is no longer a prediction. In other words, $B$ cannot be such a retrodiction where the retrodicted event $A$ influences its retrospective prediction $B$.) In symbols:

$$
A \nrightarrow B
$$


To this, the clause "without in any way disturbing a system" adds that, reversely, nor does the act of prediction influence the measurement outcome to be predicted. That is:

$$
B \nrightarrow A
$$

We have already seen what the consequent of the RC-"there exists an element of physical reality corresponding to this physical quantity"-is supposed to mean. What it means, as is clear from the explication of Goldstein et al. (2011), is that the outcome of the measurement in question is predetermined. In other words, there exists an event $\alpha$ determining that outcome $A$ will occur upon performing measurement $a$. Now, what exactly do we mean by "determine"? What we want to express is that $\alpha$ is a "sufficient cause" of $A$, given that $a$ is performed. In terms of our framework, this involves the following ingredients. First,

$$
\alpha \rightarrow A
$$

that is "determining" is a causal relation. ${ }^{21}$ Second,

$$
p(A \mid a \wedge \alpha)=1
$$

that is, whenever the "sufficient cause" $\alpha$ occurs, then the effect $A$ also occurs, given some background conditions provided here by the performance of $a$. Third, the correlation (23) of the "cause" and the "effect" must be a consequence of their causal connection (22), and not be brought about by any other causal means. Such an alternative causal means could in principle be a common causal connection of $\alpha$ and $A$ (that may exists besides their direct causal connection). So when we say that $\alpha$ determines $A$, we will assume that there exists no event $C$ such that both $C \rightarrow A$ and $C \rightarrow \alpha$ hold. For notational convenience, we introduce $\alpha \rightsquigarrow A$ for $\alpha \rightarrow A$ in the absence of common causal connection between $\alpha$ and $A .^{22}$

Putting all this together, here is what the RC states:

Reality Criterion Suppose that A is an outcome of measurement a. Assume that there is an event $B$ such that

$$
\begin{array}{r}
A \nrightarrow B \text { and } B \nrightarrow A \\
p(A \mid a \wedge B)=1
\end{array}
$$

\footnotetext{
21 We do not want to claim that interpreting determination as a causal relation is the only possibility. In the next section we will discuss an alternative interpretation, one formulated in nomological terms.

22 To illustrate the significance of this third condition, consider the following example. Two light bulbs and a switch are connected in a series circuit. When the switch is closed, both lights are on; when the switch is open, both lights are off. Since the operation of the bulbs are perfectly correlated, the state of one bulb "determines" the state of the other bulb in a statistical sense. Further, as the light bulbs are connected in series, the first bulb in the circuit has direct causal influence on the operation of the second one through influencing the charges that move towards the second bulb. (For example, were the first bulb not present, the second bulb would glow slightly brighter.) So, conditions analogous to (22) and (23) are satisfied by the states of the two light bulbs. But it is certainly not the state of one bulb that causally determines whether the other bulb is on or off; rather it is a common cause, the switch, that predetermines whether both lights are on or off.
} 
Then there exists a further event $\alpha$ such that

$$
\begin{array}{r}
\alpha \rightsquigarrow A \\
p(A \mid a \wedge \alpha)=1
\end{array}
$$

Now, let's go over to the Common Cause Principle. In its briefest formulation, the $\mathrm{CCP}$ asserts that there is no correlation without causal explanation. More precisely, whenever we observe correlation in the world, this must be either due to a direct causal connection between the correlated events, or due to a third event, a common cause, that influences both of them. The principle was first formulated by Reichenbach (1956) who characterized the common cause as a screener-off. Since its first formulation many important improvements have been made on the CCP's mathematical expression (Hofer-Szabó et al. 2013). For the sake of simplicity, here we provide a formulation essentially identical to Reichenbach's original one, with slight adjustments to the present framework:

Let $A$ and $B$ denote two arbitrary events such that

$$
\begin{array}{r}
A \nrightarrow B \text { and } B \nrightarrow A \\
p(A \wedge B) \neq p(A) p(B)
\end{array}
$$

Then there exists an event $C, a$ "common cause," such that

$$
\begin{array}{r}
C, \neg C \rightsquigarrow A, B \\
p(A \wedge B \mid C)=p(A \mid C) p(B \mid C) \\
p(A \wedge B \mid \neg C)=p(A \mid \neg C) p(B \mid \neg C)
\end{array}
$$

A special case of this general principle is when $A$ is an outcome of a measurement $a$, and $p(\cdot)=p(\cdot \mid a)$; that is, we consider a statistical subensemble of experimental runs where $a$ is always performed. This leads to the particular formulation of the CCP that we will employ:

Common Cause Principle Suppose that A is an outcome of measurement a. Assume that there is an event $B$ such that

$$
\begin{aligned}
A & \nrightarrow B \text { and } B \nrightarrow A \\
p(A \wedge B \mid a) & \neq p(A \mid a) p(B \mid a)
\end{aligned}
$$

Then there exists a further event $C$ such that

$$
\begin{aligned}
& C, \neg C \rightsquigarrow A, B \\
& p(A \wedge B \mid a \wedge C)=p(A \mid a \wedge C) p(B \mid a \wedge C) \\
& p(A \wedge B \mid a \wedge \neg C)=p(A \mid a \wedge \neg C) p(B \mid a \wedge \neg C)
\end{aligned}
$$

Proposition 1 The Reality Criterion follows from the Common Cause Principle. 
Proof Assuming that $p(A \mid a) \neq 1$, (25) obviously implies (34). Thus, since (24) is just identical with (33), the RC's antecedent implies the CCP's antecedent. We show that given the RC's antecedent, either $C$ or $\neg C$ in the CCP's consequent satisfies the conditions on $\alpha$ in the RC's consequent. (26) trivially follows from (35) both for $\alpha=C$ and $\alpha=\neg C$; hence we focus on condition (27).

(25) can be rewritten as $p(B \mid a)=p(A \wedge B \mid a)$. Applying this and the law of total probability for expressing $p(A \wedge B \mid a)$ in terms of $p(A \wedge B \mid a \wedge C)$ and $p(A \wedge B \mid a \wedge \neg C)$, while making use of (36)-(37), we receive

$$
p(B \mid a)=p(A \mid a \wedge C) p(B \mid a \wedge C) p(C \mid a)+p(A \mid a \wedge \neg C) p(B \mid a \wedge \neg C) p(\neg C \mid a)
$$

On the other hand, the law of total probability also implies

$$
p(B \mid a)=p(B \mid a \wedge C) p(C \mid a)+p(B \mid a \wedge \neg C) p(\neg C \mid a)
$$

Comparing the coefficients of $p(C \mid a)$ and $p(\neg C \mid a)$ in the two expressions for $p(B \mid a)$, we have the following options:

- If $p(B \mid a \wedge C) \neq 0$, the equality of the coefficients of $p(C \mid a)$ in (38) vs. (39) implies that $p(A \mid a \wedge C)=1$.

- If $p(B \mid a \wedge C)=0$ then $p(B \mid a \wedge \neg C) \neq 0$, otherwise $p(B \mid a)$ could not be recovered in (39) (assuming that $p(B \mid a) \neq 0$ ). In that case, the equality of the coefficients of $p(\neg C \mid a)$ in (38) vs. (39) implies that $p(A \mid a \wedge \neg C)=1$.

Thus, either $\alpha=C$ or $\alpha=\neg C$ satisfies (27). Therefore, the existence of such an $\alpha$, under the conditions in the RC's antecedent, follows from the CCP's requirement.

The basic idea behind the proved entailment is simple. Predicting with certainty the outcome of a measurement essentially means that there is perfect correlation between the result of prediction and the predicted outcome. As is well known (Hofer-Szabó et al. 2013, p. 15, Proposition 2.7), perfect correlation can only be explained, in accord with the CCP's requirement, by a deterministic common cause. Hence, the outcome must be predetermined. ${ }^{23}$

Proposition 1 can easily be extended to more general formulations of the RC's and CCP's requirements: one can have more than one pair of correlated events (for instance, due to the predicted measurement having more than one outcome); one can apply the notion of a common cause system instead of one single common cause

\footnotetext{
23 Not only the predicted outcome but also the result of prediction must therefore be predetermined by the element of reality serving as a common cause of the two. In the EPR-Bohm context this means that the element of reality in question must be localized within the intersection of the past light cones of the measurement outcomes in the two wings. This is the explanation why the element of reality, implied by the $\mathrm{RC}$ to determine the outcome in one wing, must be fixed before (or at least not be causally influenced by) the measurement choices in both wings-not only in the distant one but also in the local one. Indeed, given that the events of measurement choices $a$ and $b$ fall outside of the past light cones of the corresponding remote outcomes $B$ and $A$, respectively (which is a fact of actual experimental design; see e.g. (Redhead 1987, p. 111)), the common cause of the outcomes cannot be influenced by either of the measurement choices. See the problem raised in footnote 9 .
} 
(Hofer-Szabó et al. 2013, Sec. 7), etc. ${ }^{24}$ The main message of the result, however, is independent of these technical details. The point, we believe, is already there in the example at the beginning of this section: the basic idea behind the RC's requirement is that a successful prediction cannot be accidental, it must be brought about by causal connection. If the prediction is certain, and if the act of prediction does not disturb the predicted phenomenon, this causal connection must take the form of predetermination by a common cause.

\section{The Reality Criterion is not analytic}

It must be emphasized that even though both $B$ and $\alpha$ are characterized by the same statistical condition (25) and (27) in the RC's formula above-expressing that both $B$ and $\alpha$ "determine" the predicted measurement outcome $A$ in a statistical sense-, they are different events as they bear different, incompatible causal relations-(24) vs. (26)-to $A$. Thus, what the RC implies to exist, the element of reality predetermining the predicted outcome, is not something that logically/analytically follows from its antecedent, the possibility of predicting the outcome with certainty, without disturbing the measurement. Therefore the RC is not an analytic truth.

With this remark in mind it will be instructive to consider Tim Maudlin's (2014) claim to the contrary. Here is the crucial passage from his argument to the effect that the $\mathrm{RC}$ is an analytic truth:

[...] suppose, as the criterion demands, that I can without in any way disturbing a system predict with certainty the value of a physical quantity (for example, predict with certainty how the system will react in some experiment). Then, first, there must be some physical fact about the system that determines it will act that way. That is just to say that the physical behavior of a system depends on its physical state: if a system is certain to do something physical, then something in its physical state entails that it will do it. So determining that the system is certain to behave in some way is determining that some such physical state (element of reality) obtains. (Maudlin 2014, p. 7)

The point where this reasoning goes off course, we believe, is the conflation of two distinct notions of "determination": first, the notion that "a system is certain to do something" in the sense that we can predict with certainty how the system will behave (without disturbing it), and second, the notion that there is "something in its physical state entailing that it will do it" in the sense of there existing a sufficient cause

\footnotetext{
24 Note that Proposition 1 can also be extended to the slightly more general formulation of the RC, given by Goldstein et al. (2011), in which the "without in any way disturbing a system" clause is absent. This amounts to leaving out $B \nrightarrow A$ from (24) in the RC's antecedent. The RC thus construed can easily be shown to follow from the following version of the CCP directly capturing the idea that any correlation must be brought about either by direct causal link or by common causal connection:

Suppose that $A$ is an outcome of measurement a. Assume that there is an event $B$ such that $p(A \wedge B \mid a) \neq$ $p(A \mid a) p(B \mid a)$. Then $A \rightsquigarrow B$, or $B \rightsquigarrow A$, or there exists a further event $C$ satisfying (35)-(37).

Indeed, applying this statement to the case stipulated by the RC's antecedent without $B \nrightarrow A$, what follows is that the predicted outcome must be predetermined either by the act of prediction itself or by a common cause of the outcome and the result of prediction.
} 
predetermining its behavior. The difference here is precisely what corresponds to the distinction between $B$ and $\alpha$ in our formulation of the RC: prediction with certainty, without disturbance, means perfect correlation in the absence of direct causal connection, while causal determination means perfect correlation on account of direct causal connection. Once this crucial distinction is blurred, any statement about the relation of these two notions may come out as analytic.

Some authors have already pointed out this slip in Maudlin's argument (Lewis 2019; Glick and Boge 2019). What our analysis adds to this discussion is that we are now in a position to explicitly specify where the RC's factual content actually comes from-something those authors were contemplating. Consider Lewis's observation:

I doubt that Einstein's criterion of reality is really analytic [...] It seems perfectly conceivable that an event could be predicted with certainty even when there is nothing physical that brings that event about. [...] Indeed, Maudlin is perfectly sanguine about fundamentally probabilistic laws (e.g. in spontaneous collapse theories), according to which there is in general no physical reason why this result is obtained (as opposed to that result) when the probabilities differ from zero and one. Why should things be different when the probabilities are zero and one? (Lewis 2019, pp. 38-39)

The answer to this is now simple and clean: the reason why probability- 1 predictions are distinguished is because those predictions mean perfect correlation between the act of prediction and the predicted outcome, and a perfect correlation can only be explained, in accord with the CCP's requirement, by a deterministic common cause; hence the outcome must be predetermined. By contrast, prediction with an intermediate probability value entails non-perfect correlation that, in line with the CCP, can be accounted for by a stochastic common cause which increases the probability of the outcome in question but does not determine it. Hence, in that case, we are not entitled to infer the existence of an element of reality corresponding to that outcome.

A similar challenge to Maudlin's claim is raised by Glick and Boge:

One could be justifiably certain about a future event in an indeterministic universe, in which case there would be no prior determinant of the event [...] Suppose, for instance, that there is an oracle who has direct access to a future measurement event, the outcome of which is the result of a random, indeterministic process. One could be justifiably certain to find [the measurement outcome] without the presence of a feature of the system now that determines the outcome of this future measurement. (Glick and Boge 2019, pp. 4-5)

Indeed, the meanings of the terms "certain to find" or "determine" do not themselves make the existence of such an oracle impossible, as Maudlin's analyticity claim would suggest. Rather, it is robust principles about the causal structure of the world to which this possibility contradicts: if the oracle's prediction is indeed certain in the sense that she always gets it right, and at the same time the predicted outcome is indeed a result of an indeterministic process, then the perfect correlation of the two can only be either a consequence of some sort of retrocausal connection (contradicting that causes 
precede effects) ${ }^{25}$ or a magical coincendence without any sort of causal explanation (contradicting the CCP).

Our argument against Maudlin's analyticity claim is based on a particular translation of the RC's terms to a causal-statistical language. One can give a different interpretation to those terms, however, that does seem to support Maudlin's claim. Notice that in the EPR-Bohm case the perfect correlation of spin outcomes is predicted by a theory, quantum mechanics. One might thus relate the notions of prediction and determination to that of a theory and its laws: "predicting with certainty the value of a physical quantity" means that given certain initial/boundary conditions (in the EPR-Bohm case, the outcome of the local spin measurement), the equations of the physical theory at hand imply that the quantity in question has a certain value. On the other hand, the fact that this value is predetermined, as opposed to springing into existence by chance, means that the laws of physics, given certain initial/boundary conditions, entail this value. Now, if the laws of physics are just the equations of the physical theory at hand, then clearly the notions of "prediction with certainty" and "determination" coincide and the RC translates to an analytic truth.

The analyticity claim thus construed is not necessarily in contradiction with seeing the RC as a special case of the CCP. Indeed, in an earlier writing Maudlin seems to suggest, in line with the above interpretation of the RC, that the CCP is also analytic:

Our first condition [the screening-off condition for conditionalizing on common causes, (36)-(37)] just states that if we have taken into account every event which could serve to correlate $A$ and $B$ by causally influencing them both, then there should be no further predictable correlation between them. This condition is sometimes characterized as a demand for causal explanation for correlations, although that characterization is not quite apt. The screening-off condition allows that there be accidental correlations, due to pure chance, but requires that no such correlations be predicted by a theory. This is nearly tautologous-if a theory predicts a correlation, then that correlation cannot, according to the theory, be accidental. A nomic correlation is indicative of a causal connection-immediate or mediate-between the events, and is accounted for either by a direct causal link between them, or by a common cause of both. (Maudlin 2011, p. 82)

The added footnote is more explicit:

[...] this "principle" [the CCP] is not really a demand for a particular type of "causal" explanation, but rather a consequence of the notion of a cause. That is, such reliable correlations will validate certain counterfactual claims, and those counterfactual claims imply that there is some causal connection. (Maudlin 2011, p. 125)

That is to say: (1) Only nomic correlations-ones entailed by laws of nature-have to be brought about by causal connection, for there may exist accidental correlations. (2) Nomic correlations, on the other hand, imply counterfactual dependence, and this counterfactual dependence is what a causal connection actually consists in (on

\footnotetext{
25 Note that in this case the oracle's action doesn't actually qualify as a prediction in our causal terminology for (20) doesn't hold.
} 
the counterfactual analysis of causation). Therefore, nomic correlations, by the very meaning of "causal connection," always have a "causal explanation."

This is not the place to enter into the longstanding discussion on laws of nature, causation and their relation to scientific explanation and theories. But to defend our own view on the RC, let us close this section by raising a few difficulties that Maudlin's interpretation of the RC and the CCP may encounter in the particular case of the EPR problem:

- The EPR argument, as we formulated it in Sect. 3, can be rephrased without ever mentioning quantum theory or the laws of QM. This is because the EPR argument only refers to the minimal interpretation of QM, and hence it can be couched merely in terms of what quantum theory entails about macroscopic, observable phenomena. Indeed, imagine that someone who has never heard of QM is asked to go in the lab and register the statistics in an EPR-Bohm-type experiment. Merely on the basis of the observed statistics of macroscopic measurement events she will be able to formulate the EPR argument and conclude that those measurement outcomes must be predetermined. Therefore, anything that figures in that argument must be expressible independently from the theoretical account and laws thought to govern the observable phenomena. In particular, this must be true for the RC and the CCP.

- No doubt, counterfactual dependence is analytically tied to nomic correlation, for both of them are analytically related to laws of nature (posited by a theory). However, not every counterfactual dependency is automatically interpretable as causal connection, as the "causal explanation" of the nomic correlation in question. A case in point is Einstein's 1936 argument. ${ }^{26}$ The essence of the argument, as we reconstructed it in Sect. 4, is this. The laws of QM à la the Copenhagen interpretation-quantum state collapse-entail correlation between the choice of measurement in one wing and the quantum state of the particle in the other wing. The CCP requires casual explanation. Due to locality, there cannot be direct casual connection. Due to no-conspiracy, there cannot be common causal connection either. Thus, we have an example of a nomic correlation, entailed by the laws of Copenhagen QM, without causal explanation. That is, even though the laws of Copenhagen QM entail counterfactual dependence between the choice of measurement in one wing and the quantum state of the particle in the other wing, ${ }^{27}$ this dependency cannot be interpreted as causal connection because it doesn't satisfy certain desiderata that we want (Einstein certainly wanted) to require from causal connection on the basis of further causal principles such as locality and no-conspiracy. Notice that it is not relevant whether those causal principles are eventually maintainable or not (many believe that the main massage of the EPRBell problem is that locality is not a maintainable physical principle). Nor it is important whether the laws posited by the theory in question-the laws of QM à la the Copenhagen interpretation-are actually true in the world (the conclusion of

\footnotetext{
26 For further examples and arguments see e.g. (Lange 2016).

27 In case of stochastic laws, such as the laws of Copenhagen QM, what we may mean by counterfactual dependence is that the probability of an event counterfactually depends on the occurrence of another event. See (Lewis 1986, pp. 174-183).
} 
Einstein's argument is that they are not). The point is that the mere conceivability of an argument like Einstein's implies that it is possible to dispute whether the counterfactual dependency analytically tied to a nomic correlation really qualifies as causal connection; which means that there cannot be analytic connection between a nomic correlation and its causal explanation.

- Even if accepted that nomic correlations automatically entail causal connections in terms of which we can explain those correlations, the claim that non-nomic correlations don't require such an explanation is particularly problematic. In fact, in most cases where we apply the CCP we consider events that are not nomically related. No-conspiracy is a perfect example. Why to demand that there be no correlation between the measurement choices and the properties of the system being measured, in an EPR-Bohm experiment? This is because we believe those two things are causally independent-no direct, no common causal connection between them (on the counterfactual analysis of causation, or on any other account one may like). However, if Maudlin's claim were correct, there could always be accidental correlation, due to pure chance, between causally/nomically unrelated events, so there would be no reason to suppose that those two causally independent factorsthe choices of measurements and the system's properties-are uncorrelated. The only reason to suppose that comes from the CCP regarded as a synthetic principle that excludes precisely the sort of thing Maudlin thinks possible: accidental correlations, brought about by pure chance, not by causal connection.

\section{Discussion}

Let us now summarize the main points of our analysis from a somewhat different angle. Here is the essence of the EPR argument and Einstein's later arguments against QM, adapted to the framework of an EPR-Bohm experiment:

- EPR argument: In accord with the CCP, causal explanation for perfect correlations of spin outcomes respecting locality and no-conspiracy requires preexisting spin values in all directions.

- Einstein's later arguments: Quantum state collapse entails correlation between the choice of measurement in one wing and the quantum state of the particle in the other wing. The CCP requires casual explanation. Due to locality, there cannot be direct casual connection; due to no-conspiracy, there cannot be common causal connection either. Hence, the correlation entailed by the laws of Copenhagen QM has no causal explanation.

Given the CCP, locality, no-conspiracy, and the perfect correlations predicted by QM (à la the minimal interpretation), the EPR argument implies the existence of elements of reality-the preexisting spin values-that are not described by QM (on either of the two interpretations we considered). Hence, according the EPR argument, QM is incomplete. By contrast, Einstein's later argument shows that QM à la the Copenhagen interpretation delivers a correlation that contradicts to the $\mathrm{CCP}$, locality and no-conspiracy taken together. Hence, given the latter as premises, Einstein's argument implies that Copenhagen QM is unsound. 
From the perspective of the CCP, the structure of the two arguments is similar. The role of QM in both arguments is delivering a set of correlations we seek to explain in accord with the CCP's requirement. Locality and no-conspiracy are causal principles serve to constrain the circle of possible explanations. For the perfect correlation of spin outcomes the only possible explanation is that the spin values are predetermined in all directions. For the correlation of measurement settings and quantum states there exists no conceivable explanation that is consistent with those casual principles.

Notice, however, that there is an important difference in what the CCP is meant to require in the two cases. Einstein's later argument only makes use of a weak version of the CCP demanding that there be no correlation without some sort of causal connection. This is sufficient for Einstein's argument to work since locality and noconspiracy together ensure that there cannot be any kind of casual connection between the measurement choice in one wing and the quantum state of the particle in the other wing (hence the correlation of the two cannot possible have any sort of causal explanation). But the EPR argument employs much more of the CCP than this. Its essential ingredient is the requirement that perfect correlation, in the absence of direct causal connection, can only be explained by a deterministic common cause-something that Einstein's argument never invokes. ${ }^{28}$ It is this crucial consequence of the CCP, without which there is no incompleteness argument, that we came to call the Criterion of Reality after EPR.

Since its very first formulation, the issue of exactly what presuppositions go into Bell's no-go theorem has been subject to intense debate. Because the EPR argument was part of Bell's Bell (1964) reasoning, the same question has been asked with regard to the presuppositions of the EPR argument itself. In particular, it has been suggested, and intensely discussed recently, that some sort of "realism" is among the assumptions of the EPR argument and Bell's theorem. ${ }^{29}$ What advocates of such a view mean by realism varies from the general epistemological doctrine of scientific/metaphysical realism to more specific notions such as counterfactual definiteness or hidden variables. This is not the place to enter into this discussion. However, we would like to point out one important consequence of our thesis for this debate: as long as the "realism" assumption is meant to be related with the Reality Criterion, as EPR formulated it, this assumption is just tantamount to the Principle of the Common Cause. In other words, those who want to get around Bell's theorem by giving up "realism," as encapsulated in the $\mathrm{RC}$, those in fact deny a metaphysical principle that relates statistical regularities with the underlying casual structure of the world. This principle, as we argued, is synthetic and hence it can be subject to reevaluation. ${ }^{30}$ But it has to do with realism only to the extent to which talk about causation and causally-statistically related events presupposes realism.

\footnotetext{
28 That is, the weaker version of the CCP only refers to (33)-(35), while the stronger version essentially makes use of (36)-(37), the fact that the common cause is characterized as a screener-off.

29 See e.g. (Norsen 2007; Goldstein et al. 2011; Maudlin 2014; Werner 2014; Żukowski and Brukner 2014; Tumulka 2016; Lewis 2019)

${ }^{30}$ For possible modifications of the CCP in light of the EPR-Bell problem see (Szabó 2000; Hofer-Szabó and Vecsernyés 2012; Hofer-Szabó et al. 2013; Cavalcanti and Lal 2014).
} 
Acknowledgements This work has been supported by the National Research, Development and Innovation Office, K-115593 and K-134275, by the Ernst Mach grant, by the Alexander von Humboldt-Stiftung, and by the Hungarian Eötvös Fellowship. We wish to thank Carl Hoefer for valuable feedback.

Funding Open access funding provided by Eötvös Loránd University.

Open Access This article is licensed under a Creative Commons Attribution 4.0 International License, which permits use, sharing, adaptation, distribution and reproduction in any medium or format, as long as you give appropriate credit to the original author(s) and the source, provide a link to the Creative Commons licence, and indicate if changes were made. The images or other third party material in this article are included in the article's Creative Commons licence, unless indicated otherwise in a credit line to the material. If material is not included in the article's Creative Commons licence and your intended use is not permitted by statutory regulation or exceeds the permitted use, you will need to obtain permission directly from the copyright holder. To view a copy of this licence, visit http://creativecommons.org/licenses/by/4.0/.

\section{References}

Bell, J. S. (1964). On the Einsten-Podolsky-Rosen paradox. Physics 1, 195-200 (1964). Reprinted in: J. S. Bell, Speakable and unspeakable in quantum mechanics, (Cambridge: Cambridge University Press, 2004).

Cavalcanti, G., \& Lal, R. (2014). On modifications of Reichenbach's principle of common cause in light of Bell's theorem. J. Phys. A: Math. Theor., 47, 424010.

Einstein, A. (1936). Physik und Realität. Journal of the Franklin Institute, 221, 313-347 (1936). Translated as Physics and Reality. in Ideas and opinions, 290-323 (New York: Crown Publishing Co., 1954).

Einstein, A. (1948). Autobiographical Notes. In P. A. Schilpp (Ed.), Albert Einstein, Philosopher-Scientist. La Salle, Illinois: Open Court.

Einstein, A., Podolsky, B., \& Rosen, N. (1935). Can quantum-mechanical description of physical reality be considered complete? Physical Review, 47, 777-780.

Faye, J. (2019). Copenhagen interpretation of quantum mechanics. In: Edward N. Zalta (ed.), The Stanford Encyclopedia of Philosophy https://plato.stanford.edu/archives/win2019/entries/qm-copenhagen/.

Fine, A. (1996). The shaky game, einstein, realism and the quantum theory. Chicago: University of Chicago Press.

Fuchs, C. A., Mermin, N. D., \& Schack, R. (2014). An Introduction to QBism with an Application to the Locality of Quantum Mechanics. American Journal of Physics, 82, 749.

Gillies, D. (2000). Philosophical theories of probability. London/New York: Routledge.

Glick, D., \& Boge, F. J. (2019). Is the reality criterion analytic? Erkenntnis. https://doi.org/10.1007/s10670019-00163-W

Goldstein, S. et al. (2011). Bell's theorem. Scholarpedia,6(10), 8378, http://www.scholarpedia.org/article/ Bell's_theorem (2011).

Hájek, A., \& Bub, J. (1992). EPR. Foundations of Physics, 22, 313-331.

Harrigan, N., \& Spekkens, R. W. (2010). Einstein, incompleteness, and the epistemic view of quantum states. Foundations of Physics, 40, 125-157.

Healey, R. (2017). The quantum revolution in philosophy. Oxford: Oxford University Press.

Hoefer, C. (2019). Chance in the world: A humean guide to objective chance. Oxford: Oxford University Press.

Hofer-Szabó, G., Rédei, M., \& Szabó, L. E. (2013). The principle of the common cause. Cambridge: Cambridge University Press.

Hofer-Szabó, G., \& Vecsernyés, P. (2012). Noncommuting local common causes for correlations violating the Clauser-Horne inequality. Journal of Mathematical Physics, 53, 122301.

Howard, D. (1985). Einstein on locality and separability. Studies in History and Philosophy of Science Part A, 16, 171-201.

Howard, D. A., \& Giovanelli, M. (2019). Einstein's Philosophy of Science. In: Edward N. Zalta (ed.), The Stanford Encyclopedia of Philosophy, https://plato.stanford.edu/archives/fall2019/entries/einsteinphilscience/. 
Lange, M. (2016). Because without cause: Non-Causal explanations in science and mathematics. Oxford: Oxford University Press.

Lehner, C. (2014). Einstein's realism and his critique of quantum mechanics. In M. Janssen \& C. Lehner (Eds.), The Cambridge companion to Einstein. Cambridge: Cambridge University Press.

Lewis, D. (1986). Collected Papers (Vol. II). Oxford: Oxford University Press.

Lewis, P. J. (2019). Bell's theorem, realism, and locality. In A. Cordero (Ed.), Philosophers look at quantum mechanics. Cham: Springer.

Maudlin, T. (2011). Quantum non-locality and relativity: Metaphysical intimations of modern physics (3rd ed.). New York: Wiley-Blackwell.

Maudlin, T. (2014). What Bell did. Journal of Physics A: Mathematical and Theoretical, 47, 424010.

Maudlin, T. (2019). Philosophy of Physics: Quantum Theory. NJ: Princeton University Press.

Norsen, T. (2007). Against Realism. Foundations of Physics, 37(3), 311-340.

Norton, J. D. (2018). Einstein for Everyone, https://www.pitt.edu/ jdnorton/teaching/HPS_0410/chapters/ index.html.

Putnam, H. (1979). Philosophy of Logic. In: Mathematics Matter and Method: Philosophical Papers, Volume 1, 2nd edition, (Cambridge: Cambridge University Press, 1979).

Quine, W.V. (1980). On What There Is. In: From a Logical Point of View, 2nd edition, (Cambridge, MA: Harvard University Press, 1980).

Redhead, M. (1987). Incompleteness, nonlocality, and realism. Oxford: Clarendon Press.

Reichenbach, H. (1956). The direction of time. Berkeley: University of Los Angeles Press.

Szabó, L. E. (1995). Is quantum mechanics compatible with a deterministic universe? Two interpretations of quantum probabilities. Foundations of Physics Letters, 8, 421.

Szabó, L. E. (2000). Attempt to Resolve the EPR-Bell Paradox via Reichenbach's Concept of Common Cause. International Journal of Theoretical Physics, 39, 901-911.

Tumulka, R. (2016). The Assumptions of Bell's Proof. In M. Bell \& S. Gao (Eds.), Quantum nonlocality and reality: 50 Years of Bell's Theorem. Cambridge: Cambridge University Press.

Uffink, J. (2020). Schrödinger's reaction to the EPR paper. In: M. Hemmo and O. Shenker (eds.), Quantum, Probability, Logic: The Work and Influence of Itamar Pitowsky, (Springer, 2020).

Werner, R. F. (2014). Comment on 'What Bell did'. Journal of Physics A: Mathematical and Theoretical, 47,424010 .

Żukowski, M., \& Brukner, C. (2014). Quantum non-locality-it ain't necessarily so. Journal of Physics A: Mathematical and Theoretical, 47, 424010.

Publisher's Note Springer Nature remains neutral with regard to jurisdictional claims in published maps and institutional affiliations. 